\title{
A procedure for wall detection in [18F]FDG positron emission tomography heart studies
}

C. Landoni, V. Bettinardi, G. Lucignani, M.C. Gilardi, G. Striano, F. Fazio

INB CNR, Department of Nuclear Medicine, University of Milan Institute H San Raffaele, Via Olgettina 60, I-20132, Milan, Italy

Eur J Nucl Med (1996) 23: 18-24

Because of an unfortunate printing error, some of the mathematical symbols were omitted in Eq. 1 on p. 19 of the above article. Equation 1 is reprinted below:

$K\left(\sum_{i=1}^{12}[\mathrm{DY} \cdot \mathrm{scn}]\right)+[\mathrm{TR} \cdot \mathrm{scn}]=[\mathrm{TRDY} \cdot \mathrm{scn}]$ 